Zielińska Martyna, Zarankiewicz Natalia, Kuchnicka Aleksandra, Kosz Katarzyna, Cisel Bogumila. Pregnancy-associated breast cancer - diagnosis, treatment and outcomes for women and their offspring. Journal of Education, Health and Sport. 2020;10(8):251-259. eISSN 2391-8306. DOI http://dx.doi.org/10.12775/JEHS.2020.10.08.029

https://apcz.umk.pl/czasopisma/index.php/JEHS/article/view/JEHS.2020.10.08.029

https://zenodo.org/record/3992401

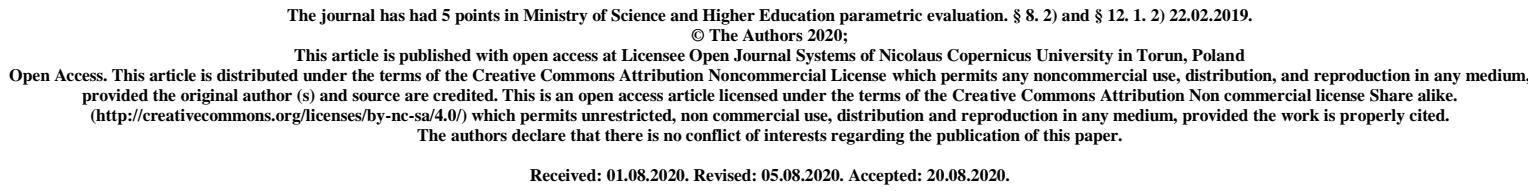

\title{
Pregnancy-associated breast cancer - diagnosis, treatment and outcomes for women and their offspring
}

\section{Martyna Maria Zielińska, Natalia Zarankiewicz, Aleksandra Kuchnicka, Katarzyna Kosz, Bogumila Cisel}

Martyna Maria Zielińska

ORCID iD https://orcid.org/0000-0001-8886-8274

Affiliation Students' Scientific Association, Oncological Surgery Department, Medical University, Lublin Country Poland

Bio Statement

Principal contact for editorial correspondence.

Natalia Zarankiewicz

ORCID iD https://orcid.org/0000-0001-9203-3376

Affiliation Students' Scientific Association, Oncological Surgery Department, Medical University, Lublin Country Poland

Bio Statement

Aleksandra Kuchnicka

ORCID iD https://orcid.org/0000-0001-8481-7592

Affiliation Students' Scientific Association, Oncological Surgery Department, Medical University, Lublin Country Poland

Bio Statement 
Katarzyna Kosz

ORCID iD https://orcid.org/0000-0001-6848-0598

Affiliation Students' Scientific Association, Oncological Surgery Department, Medical University, Lublin

Country Poland

Bio Statement

Bogumiła Ciseł

ORCID iD https://orcid.org/0000-0001-6970-1698

Affiliation Oncological Surgery Department, Independent Public Teaching Hospital No. 1, Lublin;

Medical University, Lublin

Country Poland

Bio Statement Assistant/MD, PhD (Department of Surgical Oncology)

\section{ABSTRACT}

Introduction: Pregnancy-associated breast cancer (PABC) is the most common malignancy in pregnant women with an incidence of 1 in 3-10 thousand pregnancies. It is mostly defined as breast cancer diagnosed during pregnancy or within 1 year postpartum. The number of PABCs is going to raise due to the trend of delaying childbearing.

Purpose: This review summarizes data on the occurrence and characteristics of PABC. The analysis comprises diagnosis and proper treatment of $\mathrm{BC}$ during pregnancy. Prognosis of women and their offspring are emphasized.

Material and methods: The review is based on publications mainly from 2010 to 2019 and 5 articles from 1994-2008 collected on the PubMed.

Results: The sensitivity of breast ultrasonography (USG) in pregnancy is $70-100 \%$ and it is considered as safe for the fetus. Staging evaluation in pregnant women consists of chest $\mathrm{x}$-ray, liver USG and non-contrast bone MRI. $71-88 \%$ of PABCs are invasive ductal carcinomas. Treatment consists of breast surgery regardless of time and optionally, chemotherapy from the $2^{\text {nd }}$ trimester. Radiotherapy, tamoxifen and trastuzumab are contraindicated in PABC. Therapeutic abortion does not improve oncological outcome. Overall prognosis is similar for both pregnant and non-pregnant patients with similar type and stage of $\mathrm{BC}$. The rate of congenital malformations is $1,3 \%$ for both children of $\mathrm{PABC}$ patients treated with chemotherapy and born from women without chemotherapeutic treatment. The most frequent obstetrical complication of PABC treatment is preterm delivery.

Conclusions: Pregnancy does not impact oncologic outcome, if the treatment is appropriate. PABC patients have survival rates consistent with the stage of disease. Proper treatment of PABC does not increase the risk of congenital malformations and is relatively safe for the fetus.

Key words: pregnancy-associated breast cancer; breast cancer; prognosis; pregnancy; breast cancer treatment 


\section{INTRODUCTION}

Pregnancy-associated breast cancer $(\mathrm{PABC})$ is an increasing problem worldwide. The classical definition includes cases of breast cancer (BC) diagnosed in any time of pregnancy and those diagnosed during the first year postpartum. However, some authors suggest extension of this definition to cases diagnosed within 5 or even up to 15 years after delivery [1]. Pregnancy is deemed to have twofold impact on BC risk, which was outlined for the first time by Lambe in 1994. According to the report, first 15 years after delivery increase the risk of developing $\mathrm{BC}$, while subsequent years are associated with protective impact [2]. PABC is rare, although it is the most common malignancy in pregnant women with estimated frequency of 1 in 3-10 thousand pregnancies. PABCs account for almost $16 \%$ of all BC cases in women under the age of 35 [3]. The median age of diagnosis is 33 years and 21 weeks of gestational age. Due to the trend of delaying childbearing the appearance of BCs in pregnancies is going to raise in following years [4].

\section{PURPOSE}

The aim of this review is to summarize available data on diagnosis, treatment and outcomes in women suffering from pregnancy-associated breast cancer. The influence of BC therapy on fetal and neonatal outcomes will be emphasized. While preparing this review, publications mainly from 2010-2019 and 5 articles from 1994-2008 were included in the analysis. The data was collected in the PubMed.

\section{DESCRIPTION OF THE STATE OF KNOWLEDGE}

\section{Diagnosis}

During the time of pregnancy, breast tissue undergoes physiological changes. Glandular tissue proliferates and it results in density and volume increase of breast. These changes impede precise self-examination and clinical examination of breasts. This may be the main reason of delayed diagnosis of PABC. It is estimated that the time of delay is 5-10 months in pregnant women in comparison to 1-4 months in non-pregnant ones [4]. The most common clinical presentation of PABC in the moment of diagnosis is suspicious, painless palpable mass in breast [5]. Every mass palpable for more than 2 weeks needs further examination [4].

First diagnostic step in PABC is breast ultrasonography (USG). It enables differentiation of benign and malignant tumors in pregnant women [6]. The sensitivity of USG in pregnancy is $70-100 \%$ and it is higher than sensitivity of mammography, due to increased density of breasts during pregnancy [5]. Moreover, ultrasonography allows for core biopsy under local anesthesia without endangering fetus [4].

If a mass is more suspicious, it is recommended to perform mammography [5]. This examination allows to evaluate whether any additional tumors are existing. It should be performed with proper abdominal protection in order to protect fetus, although a fetal radiation exposure is limited [4]. The sensitivity of mammography among pregnant women is about $70 \%$ with a range from 25 to even $90 \%$ in various studies $[4,7,8]$.

Breast magnetic resonance (MRI) with gadolinium should be avoided in diagnosis of PABC. A gadolinium has a detrimental effect on fetal development. However, MRI without contrast can be performed instead of scintigraphy in patients requiring skeletal system assessment [9]. 
Novel paramagnetic contrast agents such as gadobenate dimeglumine and gadoterate meglumine are approved in Europe for use in particular cases of pregnant women [7]. Nevertheless, novel agents require further investigation on their safety and efficacy.

The final diagnosis is based on a result of histopathological examination of a specimen obtained during biopsy [5]. It is estimated that around $80 \%$ of breast biopsies results are not malignant in pregnant women [4].

Staging evaluation of breast cancer is modified in pregnant women. Standard procedures in non-PABC include computed tomography (CT) of chest and abdomen and scintigraphy. In $\mathrm{PABC}$ a fetal risk is taken into account. Standard staging procedures are associated with high radiation dose for the fetus. In this regard CT and scintigraphy should be avoided in PABC [5]. If staging is necessary, other methods should be performed. It is recommended to perform chest X-ray with uterine shielding, liver ultrasonography and bone MRI without contrast [10]. An accurate assessment of nodal status is crucial due to higher risk of nodal metastasis in PABC patients and proper selection of the best possible treatment. Nodal evaluation should start with an ultrasound followed by fine needle aspiration biopsy (if required) [5]. Sentinel lymph node biopsy (SLNB) is an approved method of axillary evaluation in PABC patients with negative nodal status. Some researchers tested an influence of technetium (99-Tc) used during SLNB on fetus. No explicit danger for the fetus was observed. Using blue dyes (lymphazurin, isosulfan blue) should be avoided due to the risk of anaphylaxis. SLNB is generally safe during pregnancy and can be performed [7,11].

\section{Tumor characteristics}

The main histological type among PABC patients is invasive ductal carcinoma with a rate of $71-88 \%[3,4]$. The mean age of diagnosis is $30-38$ years [3]. Women with PABC are younger than non-PABC patients (average age 34,9 years vs. 38,5 years) [12]. PABC is more frequently diagnosed postpartum than during pregnancy $[3,13]$. In comparison to age-matched non-pregnant women with $\mathrm{BC}, \mathrm{PABC}$ tumors are characterized by more advanced stages, larger size, higher rate of G3 tumors and nodal involvement. Patients with PABC have also less frequent expression of estrogen receptors (ER) and progesterone receptors (PR) [1,3$5,13]$. It is also estimated that inflammatory breast cancer is more often diagnosed in PABC cases than in non-PABC [3].

\section{Treatment of PABC}

Treatment of PABC should be carried out with the standards of therapy of this cancer in the general patient population, taking into account the specificity of anticancer therapy during pregnancy. The essential therapeutic option in patients suffering from pregnancy-associated breast cancer in the first trimester is surgery. Radical surgery should be performed, if possible. Various authors agree that breast and axillary surgery can be safely performed in each trimester of pregnancy $[4,5,7]$. However, due to the increased risk of spontaneous abortion during the first trimester, it is reasonable to postpone surgery to the second trimester. After 12 weeks of pregnancy, the risk of miscarriage is minimal [14,15]. The decision on a type of operation should be considered individually. PABC is associated with high risk of local recurrence therefore radical mastectomy prevails over breast conserving surgery [13]. Radical modified mastectomy is recommended in first and second trimester of pregnancy [16]. 
Breast conserving surgery requires further radiotherapy, which is contraindicated during pregnancy [4,5]. It can be performed in the third trimester with radiotherapy deferred until delivery. If lumpectomy is planned at a distant time from delivery, it should be complemented by neoadjuvant or adjuvant chemotherapy. It is advised against performing lumpectomy in the first trimester if systemic therapy is not planned, due to negative impact on outcomes [14]. Breast reconstruction after mastectomy during pregnancy is considered feasible both immediately and delayed to postpartum period. It is preferred to use implants for immediate reconstruction. Due to possible difficulties of achieving symmetry between breasts because of breast engorgement during pregnancy, a deferment of reconstruction should be taken into consideration [5,7]. Nevertheless, the results of the study conducted by Lohsiriwat et al. suggest that intrapartum reconstruction is available. No serious adverse events such as hematoma or flap necrosis were reported. Moreover, 12 of 13 women who underwent immediate reconstruction using tissue expander or implant, continued their pregnancy [17].

Radiotherapy is strongly contraindicated during pregnancy, because of increased risk of disruption to organogenesis and fetal malformations [18]. It is recommended not to exceed 12 weeks pause between surgery and radiotherapy. A longer interval results in an increased risk of local relapse [19]. Some authors suggest that using proper abdominal shielding may reduce a radiation dose to fetus and avoid detrimental effects on the fetus [4,7]. Relevant research is required to evaluate whether benefits of breast irradiation during pregnancy are worth the risk. Chemotherapy is another therapeutic option for PABC patients. The use of cytostatic agents depends on the trimester of gestation. It is recommended to avoid chemotherapy in the first trimester, due to high teratogenic potential of chemotherapeutic drugs in this period of time. When a gestational age reaches 14 weeks, chemotherapy is assumed to be safe for the developing fetus [5]. The risk of fetal malformation is the highest in the first trimester with a range of $14-19 \%$ in contrary to $1,3 \%$ risk during the second and third trimesters [20]. In view of the foregoing, chemotherapy is recommended from the second trimester onwards. It is thought that chemotherapy treatment should be based on the same protocols in both PABC and not pregnant patients. In patients with pregnancy-associated breast cancer the scheme consisting of 5-fluorouracil, doxorubicin and cyclophosphamide is confirmed to be safe $[21,22]$. If tumors do not respond to anthracyclines-based regimens, the second option are taxanes, especially paclitaxel. However, the routine use of taxanes is not recommended in PABC treatment because of the insufficient data on safety profile of these agents [4,23]. There is no evidence that chemotherapy dose reduction is beneficial or harmful for the fetus. In turn, dose reduction may have a negative impact on oncological outcomes of women [5]. It is advisable to discontinue administering chemotherapeutic agents after 35th week of gestation or at least for 2 weeks before the due date in order to avoid hematological complications during labor in mother and child [4,6].

Endocrine therapy with tamoxifen during pregnancy is associated with $20 \%$ risk of congenital defects [14]. Other severe incidents such as spontaneous abortions and fetal demise have been noted [24]. Tamoxifen is contraindicated in breast cancer treatment in pregnant women and should be delayed until birth [4,5].

A study on trastuzumab administration during gestation showed that most of newborns of PABC patients treated with anti-HER2 therapy had mild to severe renal disease, pulmonary disease or infections at birth [14]. 
Poor neonatal outcomes arise out of oligohydramnios, anhydramnios, fetal heart failure, renal failure and respiratory insufficiency [4-6]. Trastuzumab is believed to be as effective when given 6 months after chemotherapy intrapartum. In this regard, trastuzumab should not be administered during pregnancy [14].

\section{Pregnancy termination}

It is considered that therapeutic abortion does not improve oncological outcomes. Maternal survival is not dependent upon pregnancy termination and depends solely on appropriate anticancer therapy. The decision on abortion has to be individual and based on the risk of possible progression of malignancy or fetal harm related to oncologic therapy [25].

\section{Neonatal outcomes}

Minimal detrimental impact on the fetus related to the use of chemotherapeutic agents is related to lowered level of these agents within the fetal system as opposed to maternal system [5,21]. The long-term follow-up conducted by Aviles et al. on children born from women treated with chemotherapy due to hematological malignancies showed no neurocognitive, physical nor psychological effects among these children [26]. The most frequent obstetrical complication of PABC treatment is preterm delivery. It is estimated that breast cancer is a factor of an even 5-fold increased risk of premature labor in comparison to pregnancies in healthy women $(30,05 \%$ vs. $7,21 \%, \mathrm{p}<0.0001)$. However, it is under consideration whether this increased ratio of preterm labors is associated only with the anticancer treatment, the cancer itself or with the iatrogenic induction of preterm deliveries in order to undergo more aggressive treatment [27]. Another research showed the premature deliveries incidence rate of $54,6 \%$ in comparison to $50 \%$ in general population based on The European Registry. The average time of delivery was 35,7 +/- 3,2 weeks [23]. Pregnancies in women suffering from breast cancer have also a twofold greater risk of preterm premature rupture of membranes [27]. There is no difference in the rate of congenital malformations between the offspring born from women who underwent chemotherapy in pregnancy and women not treated with chemotherapeutic agents and it amounts to $1,3 \%$ [6]. The offspring of PABC patients treated appropriately are not under a higher risk of congenital anomalies, intrauterine growth restriction and intrauterine fetal demise [27]. Nevertheless, it should be pointed out that obstetric complications observed in several studies are not only preterm labor and preterm rupture of membranes, but also severe intrauterine growth restriction, oligohydramnios, anemia, dyspnea, pneumonia, preeclampsia, respiratory distress syndrome and necrotizing enterocolitis in newborn [6, 23, 27].

\section{Prognosis}

Prognosis of PABC patients is very important issue discussed by various researchers. The data on this subject is inconclusive. Most recent data suggest that overall prognosis does not differ between age-matched pregnant and non-pregnant patients with similar type and stage of breast cancer [5,28]. The results of a study carried out by Genin et al. showed no significant differences in overall survival (OS), disease free survival (DFS) and distant relapse rates between PABC patients and non-pregnant controls [13]. 
Another study showed similar 5-year survival rate between both pregnant and non-pregnant patients treated with 5-fluorouracil, adriamycin and cyclophosphamide (77\% vs. $71 \%, \mathrm{p}=$ 0.046) [29]. Other authors suggest worse prognosis of PABC patients due to young age and delayed diagnosis [30]. One more poor prognostic factor is more frequent occurrence of T3T4 tumors and negative hormonal status. It is also believed that tumors in pregnant women have the same poor prognosis regardless of their advance stage as not pregnant women with T3-T4 tumors [13]. It has been proven that local recurrence occurs more often in pregnancyassociated breast cancer patients rather than non-pregnant controls [13,28]. Moreover, patients diagnosed with BC postpartum have inferior outcomes, especially when diagnosed within 1 year after delivery. In a study conducted by Van Den Rul et al. BC patients diagnosed within 1 year after labor had decreased 5-year DFS (53\%) and OS (60\%) rates in comparison to not pregnant controls (DFS $=68 \%, \mathrm{OS}=84 \%$ ) [31]. Lowest survival rate of $38 \%$ is found in $\mathrm{BC}$ patients diagnosed during 12 months postpartum and increases in a later period reaching 51$60 \%$ in 13-48 months postpartum, in comparison to nulliparous women with a survival rate of $65 \%$ [12]. Increased rates of distant metastases in liver and brain were also observed in postpartum breast cancer [32]. There are no contraindications for becoming pregnant after surviving breast cancer in young patients. It is recommended to discontinue taking tamoxifen for at least 2 months before attempting pregnancy [5].

\section{SUMMARY}

Pregnancy-associated breast cancer is a significant challenge in oncology nowadays. Besides maternal benefits from anticancer treatment, fetal well-being should be carefully evaluated. The therapy plan should be based on the decision of the multidisciplinary team. Treatment of breast cancer is trimester-dependent in pregnant women. To the best of our knowledge, gestation does not impact oncologic outcome, if the treatment is appropriate. Breast surgery remains the essential therapeutic option in pregnant patients, with possible chemotherapy from the second trimester. Radiotherapy, anti-HER2 therapy and hormonal therapy should be avoided until labor. There is no significant risk of serious detrimental effects on fetus. The prognosis of the offspring of PABC patients is good and pregnancy termination is not recommended as it does not improve maternal outcomes. Pregnant women suffering from BC have survival rates consistent with the stage of disease.

\section{REFERENCES}

1. Pugh AM, Giannini CM, Pinney SM, Hanseman DJ, Shaughnessy EA, Lewis JD. Characteristics and diagnosis of pregnancy and lactation associated breast cancer: Analysis of a self-reported regional registry. Am J Surg. 2018;216(4):809-812. doi: 10.1016/j.amjsurg.2018.07.060.

2. Lambe M, Hsieh CC, Trichopoulos D, Ekbom A, Pavia M, Adami HO. Transient increase in the risk of breast cancer after giving birth. N Engl J Med. 1994;331:5-9.

3. Ruiz R, Herrero C, Strasser-Weippl K, Touya D, St Louis J, Bukowski A et al. Epidemiology and pathophysiology of pregnancy-associated breast cancer: A review. Breast. 2017;35:136-141. doi: 10.1016/j.breast.2017.07.008. 
4. Martinez MT, Bermejo B, Hernando C, Gambardella V, Cejalvo JM, Lluch A. Breast cancer in pregnant patients: A review of the literature. Eur. J. Obstet. Gynecol. Reprod. Biol. 2018;230:222-227. doi.org/10.1016/j.ejogrb.2018.04.029.

5. Becker S. Breast cancer in pregnancy: A brief clinical review. Best Pract Res Clin Obstet Gynaecol. 2016;33:79-85. doi: 10.1016/j.bpobgyn.2015.10.013.

6. Córdoba O, Llurba E, Saura C, Rubio I, Ferrer Q, Cortés J et al. Multidisciplinary approach to breast cancer diagnosed during pregnancy: maternal and neonatal outcomes. Breast. 2013;22(4):515-9. doi: 10.1016/j.breast.2012.10.005.

7. Cordeiro C, Gemignani M. Breast Cancer in Pregnancy: Avoiding Fetal Harm When Maternal Treatment Is Necessary. Breast J. 2017;23(2):200-205. doi:10.1111/tbj.12780.

8. Langer A, Mohallem M, Stevens D, Rouzier R, Lerebours F, Cherel P. A singleinstitution study of 117 pregnancy-associated breast cancers (PABC): Presentation, imaging, clinicopathological data and outcome. Diagn Interv Imaging. 2014;95(4):435-41. doi: 10.1016/j.diii.2013.12.021.

9. Kanal E, Barkovich AJ, Bell C, et al. ACR guidance document for safe MR practices: 2007. A J Roentgenol. 2007;188:1447-1474. doi: 10.2214/AJR.06.1616.

10. Amant F, Loibl S, Neven P, Van Calsteren K. Breast cancer in pregnancy. Lancet Oncol. 2012;379:570-579. doi: 10.1016/S1470-2045(11)70363.-1.

11. Gropper AB, Zabicki Calvillo K, Dominici L, Troyan S, Rhei E, Economy KE et al. Sentinel Lymph Node Biopsy in Pregnant Women With Breast Cancer. Ann Surg Oncol. 2014;21(8):2506-2511. doi: 10.1245/s10434-014-3718-2.

12. Genin AS, Lesieur B, Gligorov J, Antoine M, Selleret L, Rouzier R. Pregnancyassociated Breast Cancers: Do They Differ From Other Breast Cancers in Young Women? Breast. 2012;21(4):550-555. doi: 10.1016/j.breast.2012.05.002.

13. Genin AS, Rycke Y, Stevens D, Donnadieu A, Langer A, Rouzier R et al. Association With Pregnancy Increases the Risk of Local Recurrence but Does Not Impact Overall Survival in Breast Cancer: A Case-Control Study of 87 Cases. Breast. 2016;30:222-227. doi: 10.1016/j.breast.2015.09.006.

14. Zagouri F, Sergentanis TN, Chrysikos D, Papadimitriou CA, Dimopoulos MA, Bartsch R. Trastuzumab administration during pregnancy: a systematic review and metaanalysis. Breast Cancer Res Treat. 2013;137(2):349-357. doi:10.1111/tbj.12780.

15. Grimm D, Woelber L, Trillsch F, Keller-v Amsberg G, Mahner S. Clinical management of epithelial ovarian cancer during pregnancy. Eur J Cancer. 2014;50(5):963971. doi: 10.1016/j.ejca.2013.12.020.

16. Fajdic J, Gotovac N, Hrgovic Z, Fassbender WJ. Diagnosis and therapy of gestational breast cancer: a review. Adv Med Sci. 2008;53(2):167-171. doi: 10.1111/tbj.12780

17. Lohsiriwat V, Peccatori FA, Martella S, Azim HA Jr, Sarno MA, Galimberti V et al. Immediate breast reconstruction with expander in pregnant breast cancer patients. Breast. 2013;22(5):657-660. doi: 10.1016/j.breast.2013.06.005.

18. Loibl S, Schmidt A, Gentilini O, Kaufman B, Kuhl C, Denkert C et al. Breast cancer diagnosed during pregnancy: adapting recent advances in breast cancer care for pregnant patients. JAMA Oncol. 2015;1(8):1145-1153. doi: 10.1001/jamaoncol.2015.2431 
19. Toesca A, Gentilini O, Peccatori F, Azim HA, Amant F. Locoregional treatment of breast cancer during pregnancy. Gynecol Surg. 2014;11:279-284. doi: 10.1007/s10397-0140860-6.

20. Azim Jr. HA, Del Mastro L, Scarfone G, Peccatori FA. Treatment of breast cancer during pregnancy: regimen selection, pregnancy monitoring and more. Breast. 2011;20:1-6. doi.org/10.1016/j.breast.2010.10.008

21. Amant F, Van Calsteren K, Halaska MJ, Gziri MM, Hui W, Lagae L, et al. Long-term cognitive and cardiac outcomes after prenatal exposure to chemotherapy in children aged 18 months or older: an observational study. Lancet Oncol. 2012;13(3):256-264. doi: 10.1016/S1470-2045(11)70363-1.

22. Hahn KM, Johnson PH, Gordon N. Treatment of pregnant breast cancer patients and outcomes of children exposed to chemotherapy in utero. Cancer. 2006;107(6):1219-1226. doi: $10.1002 /$ cncr.22081

23. Framarino-dei-Malatesta M, Piccioni MG, Brunelli R, Iannini I, Cascialli G, Sammartino P. Breast cancer during pregnancy: a retrospective study on obstetrical problems and survival. Eur J Obstet Gynecol Reprod Biol. 2014;173:48-52. Doi.org/10.1016/j.ejogrb.2013.11.017

24. Guidroz JA, Scott-Conner CE, Weigel RJ. Management of pregnant women with breast cancer. J Surg Oncol 2011;103:337-340. doi: 10.1002/jso.21673

25. Amant F, Brepoels L, Halaska MJ, Gziri MM, Calsteren KV. Gynaecologic cancer complicating pregnancy: an overview. Best Pract Res Clin Obstet Gynaecol. 2010;24(1):6179. doi: 10.1016/j.bpobgyn.2009.08.001

26. Aviles A, Neri N. Hematologic malignancies and pregnancy: a final report of 84 children who received chemotherapy in utero. Clin Lymphoma. 2001;2(3)173-177. doi: 10.3816/CLM.2001.n.023

27. Maor GS, Czuzoj-Schulman N, Spence AR, Abenhaim HA. Neonatal outcomes of pregnancy-associated breast cancer: Population-based study on 11 million births. Breast J. 2019;25(1):86-90. doi: 10.1111/tbj.13156

28. Peccatori FA, Azim HA. Managing pregnancy-associated breast cancer: Is more really better? Breast. 2016;30:215-216. doi: 10.1016/j.breast.2016.06.006

29. Litton JK, Warneke CL, Hahn KM, Palla SL, Kuerer HM, Perkins GH et al. Case control study of women treated with chemotherapy for breast cancer during pregnancy as compared with nonpregnant patients with breast cancer. Oncologist. 2013;18(4):369-376. doi: 10.1634/theoncologist.2012-0340

30. Azim HA, Santoro L, Russell-Edu W, Pentheroudakis G, Pavlidis N, Peccatori A. Prognosis of pregnancy-associated breast cancer: A meta-analysis of 30 studies. Cancer Treat Rev. 2012;38(7):834-842. doi: 10.1016/j.ctrv.2012.06.004

31. Van den Rul N, Han SN, Van Calsteren K, Neven P, Amant F. Postpartum breast cancer behaves differently. Facts Views Vis Obgyn. 2011;3(3):183-188.

32. Eng Lee G, Mayer EL, Partridge A. Prognosis of pregnancy-associated breast cancer. Breast Cancer Res Treat. 2017;163:417-421. doi: 10.1007/s10549-017-4224-6 